\title{
Simvastatina para la prevención de exacerbaciones en EPOC moderada a grave
}

\section{Simvastatin for the prevention of exacerbations of moderate-to-severe COPD}

Karen Navarro Pérez, MD. (1); Carlos Celis Preciado, MD. (2)

(1) Residente de primer año de Medicina Interna, Pontificia Universidad Javeriana, Hospital Universitario San Ignacio, Bogotá, Colombia.

${ }^{(2)}$ Internista, Neumólogo, Hospital Universitario San Ignacio, Pontificia Universidad Javeriana, Bogotá, Colombia.

Correspondencia: Karen Navarro Pérez, correo electrónico: kren97@hotmail.com Recibido: 12/02/14, Aprobado: 20/02/14.

\section{Referencia}

Criner GJ, Connett JE, Aaron SD, Albert RK, Bailey WC, Casaburi R, et al. COPD Clinical Reseach Network and the Canadian Institutes of Health Reseach. Simvastatin for the Prevention of Exacerbation in Moderate -to- severe COPD. N Engl J Med. 2014;370:2201-10.

\section{Pregunta}

¿Disminuye la simvastativa administrada diariamente la tasa de exacerbaciones anuales en pacientes con EPOC moderada a grave en comparación con placebo?

\section{Diseño}

Estudio prospectivo, aleatorizado, grupo paralelo, controlado con placebo. STATCOPE (del Inglés Statins in COPD Exacerbations).

\section{Periodo de seguimiento}

12, 24 y 36 meses. 641 días en promedio en el grupo de simvastatina y 639 en el grupo placebo.

\section{Lugar}

45 centros médicos, 29 en los Estados Unidos y 16 en Canadá.

\section{Pacientes}

885 pacientes entre 40 y 80 años de edad con diagnóstico de EPOC $\left(\mathrm{VEF}_{1}\right.$ menor del $80 \%$, relación $\mathrm{VEF}_{1} / \mathrm{CVF}$ menor del 70\%) e his- toria de tabaquismo de más de 10 paquetes/año que requirieron en el año previo a la aleatorización, al menos uno de los siguientes: uso de oxígeno suplementario, tratamiento con glucocorticoides o antibióticos, visita al departamento de urgencias u hospitalización por exacerbación.

Se excluyeron pacientes en tratamiento con estatinas, quienes recibieran medicamentos que contraindicaran el uso concomitante de estatinas, aquellos con enfermedad hepática activa, alcoholismo o alergia al medicamento.

Se incluyeron 430 pacientes en el grupo de simvastatina y 447 en el placebo.

\section{Intervención}

- Simvastatina $40 \mathrm{mg} /$ día vs. placebo.

\section{Resultados}

- Tras el inicio del estudio, la Administración de Drogas y Alimentos (FDA, su sigla en Inglés) expidió una alerta repecto al uso concomitante de amlodipino o altas dosis de verapamilo con simvastatina; por tanto se excluyeron 16 pacientes del grupo de simvastatina y 20 del placebo, y adicionalmente, 28 (14 en cada grupo) por diabetes. 
- Durante el estudio se reportaron en total 1.982 exacerbaciones, 965 en el grupo de simvastatina y 1.017 en el placebo (tasa de 1,36 vs. 1,39 exacerbaciones por persona/año respectivamente $(p=0,54)$.

- En cuanto al número de días para la primera exacerbación: 223 en el grupo de simvastatina en comparación con 231 días en el grupo placebo $(\mathrm{p}=0,34)$.

- 296 pacientes tuvieron más de tres exacerbaciones: 155 en el grupo placebo, 141 en el de simvastatina.

- No hubo diferencia en función pulmonar ni calidad de vida, así como tampoco en eventos adversos no fatales, excepto aquellos con compromiso del tracto gastrointestinal que fueron más frecuentes en el grupo de simvastatina (30 vs. 17 pacientes en el grupo placebo) (tabla 1).

\section{Conclusión}

La simvastatina en dosis de $40 \mathrm{mg} /$ día no reduce las tasas de exacerbación ni prolonga el tiempo para la primera exacerbación en pacientes con EPOC moderada a grave ni tiene efecto en la función pulmonar ni en la calidad de vida. Las tasas de eventos adversos fueron bajas y similares en los grupos de estudio.

\section{Comentario}

Antes de la publicación de este estudio, al menos tres revisiones sistemáticas de estudios observacionales, dos de ellas con metaanálisis, habían reportado que el empleo de estatinas en pacientes con en- fermedad pulmonar obstructiva crónica (EPOC) se asociaba con menor riesgo de mortalidad por todas las causas y respiratoria, menor frecuencia de exacerbaciones y de intubaciones relacionadas, y mejor función pulmonar y de capacidad de ejercicio (1-3).

El trabajo de Criner y cols. (4-5), es el primer estudio de asignación aleatoria diseñado para evaluar el efecto de una estatina (rosuvastatina) en el riesgo de exacerbaciones de la enfermedad. Entre las razones por las cuales el estudio no mostró un resultado concordante con la información previa se señala:

- Inclusión de pacientes con EPOC con bajo riesgo cardiovascular (mortalidad en el grupo placebo de $6 \%$, la mitad a la observada en el estudio TORCH, y reducción de las cifras de LDL tan solo del $23 \%$, menos del $36 \%-40 \%$ esperados con la dosis utilizada).

- Uso de esteroides inhalados en el 73\% de los pacientes (en comparación con un 7\% en promedio en los estudios observacionales).

- Falta de información acerca de la proporción de pacientes originalmente asignados a placebo y que recibieron estatina a lo largo del seguimiento.

- Falta de estratificación de los pacientes según cifras de proteína $\mathrm{C}$ reactiva (PCR), teniendo en cuenta que trabajos como el estudio JUPITER han reportado que el efecto de las estatinas podría estar relacionado con el grado de inflamación basal del paciente.

Por tanto, se necesita evidencia reciente acerca del empleo de estatinas como tratamiento adjunto

Tabla 1. Desenlaces en los grupos de terapia con simvastatina y placebo.

\begin{tabular}{|c|c|c|c|}
\hline Desenlace & Simvastatina & Placebo & $\mathbf{p}$ \\
\hline Número de exacerbaciones & 965 & 1017 & \\
\hline Tasa de exacerbaciones (persona/año) & $1,36 \pm 1,61$ & $1,39 \pm 1,73$ & 0,54 \\
\hline Días para la primera exacerbación & 223 & 231 & 0,34 \\
\hline Más de tres exacerbaciones & 141 & 155 & \\
\hline $\begin{array}{l}\text { Eventos adversos no fatales (número de } \\
\text { eventos serios persona/año) }\end{array}$ & 0,63 & 0,62 & $>0,20$ \\
\hline Muertes & 28 & 30 & 0,89 \\
\hline
\end{tabular}


en pacientes con EPOC, antes de concluir que dicha estrategia no es benéfica en los pacientes sin indicación cardiovascular del medicamento (4-5).

\section{Conflictos de intereses}

Los autores declaran no tener conflictos de interés.

\section{Bibliografía}

1. Dobler CC, Wong KK, Marks GB. Associations between statins and COPD: a systematic review. BMC Pulm Med. 2009;9:32.
2. Janda S, Park K, FitzGerald JM, Etminan M, Swiston J. Statins in COPD: a systematic review. Chest. 2009;136:734-43.

3. Horita N, Miyazawa N, Kojima R, Inoue M, Ishigatsubo Y, Ueda A, Kaneko T. Statins reduce all-cause mortality in chronic obstructive pulmonary disease: a systematic review and meta-analysis of observational studies. Respir Res. 2014;15:80.

4. Young RP, Hopkins RJ, Agusti A. Statins as adjunct therapy in COPD: how do we cope after STATCOPE? Thorax. 2014;69: 891-4.

5. Mancini GB, Road J. Are Statins out in the COLD? The STATCOPE Trial. Can J Cardiol. 2015;31:970-3 